\title{
Synthesis of Porous Materials and Their Microstructural Control through Ice Templating
}

\author{
Wiwut Tanthapanichakoon ${ }^{1, a}$, Hajime Tamon ${ }^{2, b}$, Kyuya Nakagawa ${ }^{3, c}$, \\ and Tawatchai Charinpanitkul ${ }^{4, \mathrm{~d}}$ \\ 1 Department of Chemical Engineering, Tokyo Institute of Technology, Tokyo 152-8550, Japan \\ 2 Department of Chemical Engineering, Kyoto University, Katsura, Kyoto 615-8510, Japan \\ 3 Research Center for Nano-Micro Structure Science and Engineering, Graduate School of Engineering, \\ University of Hyogo, 2167 Shosha, Himeji, Hyogo 671-2280, Japan \\ 4 Center of Excellence in Particle Technology, Chulalongkorn University, Bangkok, Thailand \\ E-mail: awwiwutt@chemeng.titech.ac.jp (Corresponding author), btamon@cheme.kyoto-u.ac.jp, \\ cnakagawa@eng.u-hyogo.ac.jp, ${ }^{\mathrm{d}} \mathrm{ctawat} @$ chula.ac.th
}

\begin{abstract}
Ice-templating is a simple, practical kind of template synthesis which consists of sol-gel polycondensation, unidirectional freezing of hydrosols or hydrogels, and porepreserving drying method, such as freeze drying, thereby resulting in desirable porous materials. The unidirectional freezing may be achieved by either immersion freezing or contact freezing. Freeze-dried materials contain porous microstructures that are replicas of the ice crystals formed during the unidirectional freezing process. Two similar but slightly different applications of ice-templating will be investigated here. Fabrication of macroporous foam materials by unidirectional freezing of an aqueous suspension of carbon nanotubes (CNTs) dispersed by chitosan is a concrete example of functionalization of an electroconductive foam, which is a composite material made from a polymer and CNTs. As expected, their electroconductive properties can be controlled by the fabrication method and condition. Additional examples of porous materials synthesized by icetemplating are porous microfibers and microhoneycombs of silica, titania, silica-alumina, titania-silica, and even carbon. In the preparation, the morphology and porous structure can be controlled by the freezing conditions and synthesis conditions of the corresponding hydrogels, respectively.
\end{abstract}

Keywords: Porous material, ice templating, microhoneycomb, microfiber, CNT.

ENGINEERING JOURNAL Volume 17 Issue 3

Received 3 April 2013

Accepted 3 April 2013

Published 1 July 2013

Online at http://www.engj.org/

DOI:10.4186/ej.2013.17.3.1 


\section{Introduction}

Porous materials find wide, diverse applications in a great variety of industries and our daily life, thanks to their high specific surface areas (upwards of $2,500 \mathrm{~m}^{2} / \mathrm{gram}$ ), sieving effect (down to molecular scale), excellent thermal insulation property, acoustic damping, extremely light weight (down to $20 \mathrm{~kg} / \mathrm{m}^{3}$ ), comparatively high strength, and so on. Porous materials may consist of regular crystalline or irregular amorphous, organic or inorganic, frameworks which support uniform, hierarchical, or complex porous structures. According to IUPAC nomenclature, micropores, mesopores and macropores possess pore size ranges of $0.2-2 \mathrm{~nm}, 2-50 \mathrm{~nm}$, and $50-1000 \mathrm{~nm}$, respectively. Widely used as catalysts, adsorbents and sensing probes, porous materials have recently become key materials in energy storage devices, such as lithium ion batteries and super capacitors. Micropores and small mesopores mainly provide the unique functions of porous materials, and substances that want to obtain the benefits of functionalization may gain access to these small mesopores and micropores only by passing through the macropores or large mesopores of the material. Subsequently, the transformed substances or products made at the sites of functionalization must pass through these large pores to come out of the material. Therefore the accompanying transport processes are very important, and in certain cases, they even determine the overall performance of the material itself. Depending on the application of interest, we want to synthesize porous materials with suitable, controlled microsturctures.

There is a great variety of methods to fabricate and synthesize porous materials. The present article will focus on one of the most simple and practical methods, namely, ice-templating. Two slightly different applications of ice-templating will be investigated and reported here. The first employs sol-gel polycondensation, unidirectional freezing of hydrosols or hydrogels, and pore-preserving drying method such as freeze drying, to obtain desirable porous materials. This method has successfully been employed to synthesize porous microfibers or microhoneycombs [1-4]. When hydrosols or hydrogels are dipped into cryogenic coolant at a constant rate, pseudo-steady-state freezing occurs. Ice formation grows as a pillar and the sol or the gel is concentrated between the growing ice crystals. After thawing and drying, microfibers and microhoneycombs are obtained. In the preparation, ice crystal plays the key role of template. In fact, 4 kinds of morphology of non-powder silica gels-lamella, flat fiber, microhoneycomb and polygonal fiber-can be synthesized from hydrosols and hydrogels as shown in Fig. 1 [1, 5]. More specifically, porous silica gels with 3 kinds of morphology (lamella, flat fiber and microhoneycomb) are prepared through unidirectional freezing of hydrosols. On the other hand, porous silica gels prepared by unidirectional freezing of hydrogels have 3 kinds of morphology (microhoneycomb, fiber with polygonal cross-section and powder). The morphology of silica gels prepared is mapped for the gelation time, the aging time and the delay time determined from dynamic light scattering (DLS) measurements of silica hydrosols. In short, the morphology of silica gel can readily be controlled by using the proposed maps [5].

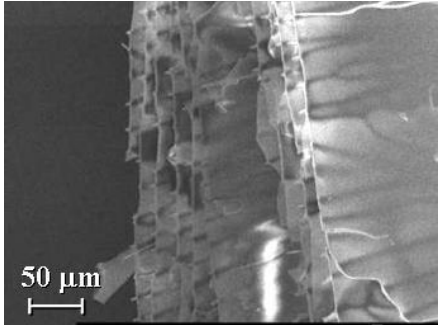

(a) Lamella

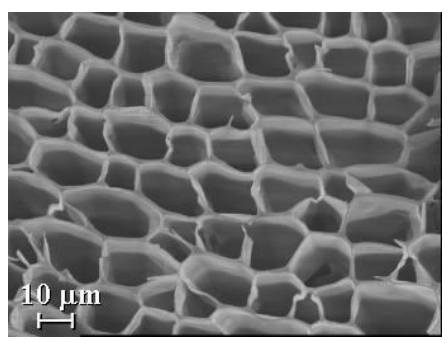

(c) Microhoneycomb

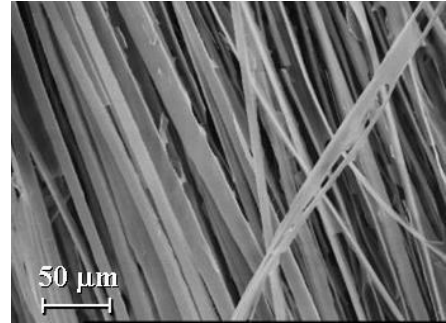

(b) Flat fiber

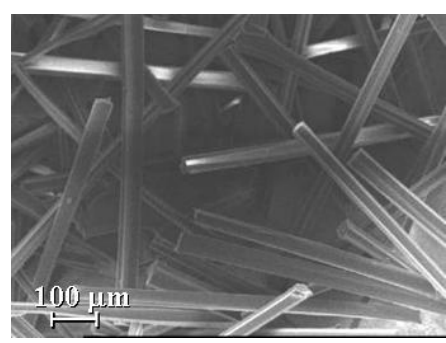

(d) Polygonal fiber

Fig. 1. Morphology of silica gels prepared by unidirectional freezing of hydrogels. 
In contrast, the typical foam is a macroporous material that comes in many shapes. The second application of ice-templating here is to prepare foam materials from carbon nanotubes (CNTs) by manipulating the accompanying solid-liquid phase separation process. Ice formation in a frozen colloidal or sol-gel system is controlled by interfacial phenomenon during solidification. In principle, the moving freezing front would reject suspended particles when the front velocity is sufficiently low, while it would engulf them when the velocity is sufficiently high. The resulting CNT foam is expected to exhibit a specific functionality derived from nanocarbons in the bulk structuralized material [6] (Fig. 2). Related studies have clearly shown that ice is an interesting tool for creating interconnected 3D microstructures in freeze-dried CNT foams [7-11].

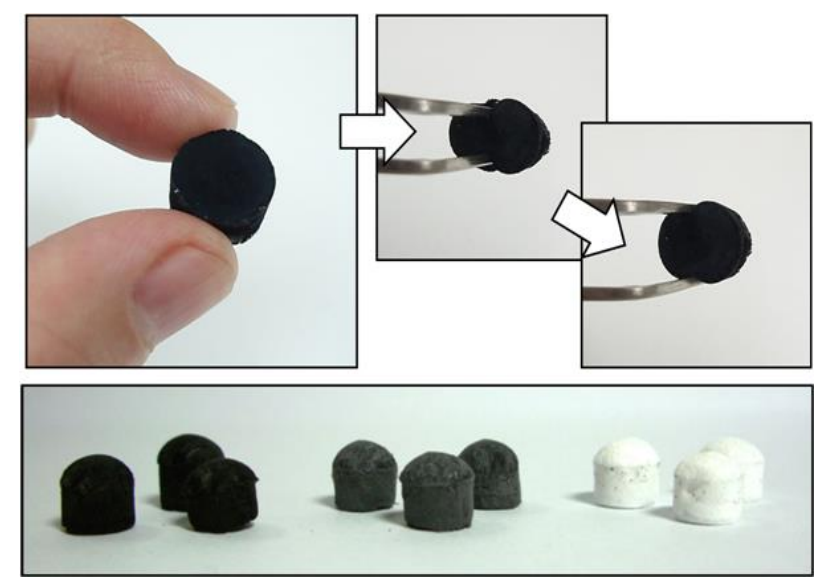

Fig. 2. Foam materials made from carbon nanotubes and titanium nanopowders.

Leroy et al. [12] demonstrated CNT foam preparation via a bubbling and subsequent freeze-drying process that enabled unique microstructure formation. Sol-gel processing was also useful for producing CNT foams with nanostructured CNT arrays [13]. To create a controlled microstructure by making use of the accompanying phase separation process during ice crystal formation, we must control the kinetics of phase transition (e.g. evaporation for the foaming process, solidification for the freezing process). In this regard, freezing would be strategically advantageous for engineering porous materials because freezing can readily be controlled by a thermal system.

The present investigation aims to demonstrate the versatility of ice-templating to produce a wide range of porous materials in a variety of morphology and shapes with the desired microstructures. What follow are the details of the two similar but distinctive applications mentioned above.

\section{Microstructure Control of Silica Gel Microhoneycombs}

The morphology of silica gel microhoneycombs (SMHs) depends on the unidirectional freezing conditions [14]. Figure 3 shows the relation of average macropore size (channel size) of SMHs $d_{\text {mac }}$ and freezing condition $\left\{v_{\mathrm{f}}\left(T_{\mathrm{r}}-T_{\mathrm{f}}\right)\right\}^{-1}$, where $v_{\mathrm{f}}[\mathrm{m} / \mathrm{s}]$ is the immersing rate of a sample into the coolant (the freezing rate), $T_{\mathrm{r}}[\mathrm{K}]$ is the room temperature and $T_{\mathrm{f}}[\mathrm{K}]$ is the freezing temperature. The values of $d_{\mathrm{mac}}$ can be correlated to $\left\{v_{\mathrm{f}}\left(T_{\mathrm{r}}-T_{\mathrm{f}}\right)\right\}^{-1}$, and $d_{\mathrm{mac}}$ increases with an increase in freezing temperature and a decrease in freezing rate. Figure 4 shows the relation of average wall thickness of SMHs $l_{\mathrm{w}}$ and $C_{\mathrm{s}} d_{\mathrm{mac}}$. Here $C_{\mathrm{s}}\left[\mathrm{mol} / \mathrm{m}^{3}\right]$ is the silica concentration of the starting solution for silica gel synthesis. The ratio of the total volume of honeycomb walls against the total volume of ice crystals should increase with an increase in $C_{\text {s }}$; i.e., the wall thickness of the SMHs is expected to increase with an increase in $C_{s}$. On the other hand, it can be easily noticed that the wall thickness of SMHs will increase with an increase in macropore size when the volume ratio of the honeycomb walls remains constant. Figures 3 and 4 indicate that the morphology of SMHs can be controlled by the unidirectional freezing conditions and the silica concentration.

SMHs prepared through this simple ice-templating method have extremely low apparent density (ca. $0.12 \mathrm{~g} / \mathrm{cm}^{3}$ ), which are similar to those of silica aerogels. On the other hand, the density of the walls of $\mathrm{SMHs}$ is about $1.2 \mathrm{~g} / \mathrm{cm}^{3}$, which is close to those of conventional silica gels. Therefore, it is expected that the conventional methods for controlling the micro-/meso-porosity of silica gels should be applicable to 
SMHs. In fact the micro-/meso-porosity of the walls of SMHs can actually be modified by adjusting the $\mathrm{pH}$ of their parent sols [2]. However, the $\mathrm{pH}$ also affects the gelation time of silica sols and the firmness of the resulting silica hydrogels. Since there exists a $\mathrm{pH}$ region where the microhoneycomb structure cannot be obtained, it is difficult to precisely control the micro-/meso-porosity of SMHs only by changing the $\mathrm{pH}$ of their parent sols. On the other hand, hydrothermal treatment after the formation of honeycomb structure is available for controlling mesoporosity of SMHs. In the hydrothermal treatment, "Ostwald ripening" proceeds; i.e., smaller nanoparticles that constitute the silica gel matrixes dissolve and reprecipitate on the surface of larger nanoparticles. It has been reported that by applying the hydrothermal treatment in a basic solution to SMHs the sizes of the mesopores inside the honeycomb walls can be varied in a wide range of less than 2 to $45 \mathrm{~nm}$ [14].

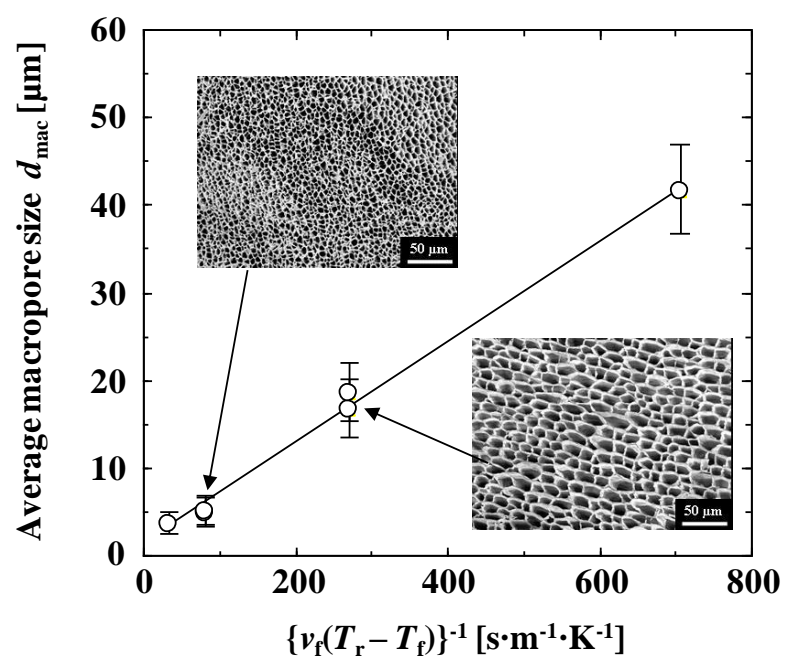

Fig. 3. Correlation of average macropore size of SMHs with the freezing condition.

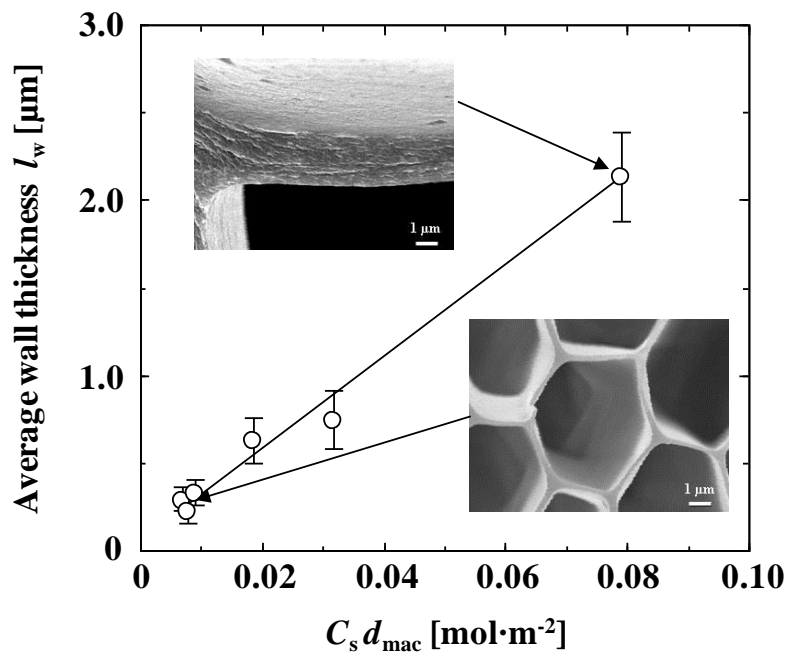

Fig. 4. Correlation of average wall thickness of SMHs with $C_{\mathrm{s}} d_{\mathrm{mac}}$.

\section{Synthesis of Various Materials with Microfiber or Microhoneycomb Morphology}

Figure 5 shows examples of SEM images of the materials prepared by applying the present ice-templating method to various materials. Titania $\left(\mathrm{TiO}_{2}\right)$ cryogel microfibers with high BET surface area are prepared through the unidirectional freezing and subsequent freeze-drying of $\mathrm{TiO}_{2}$ hydrogels, which are synthesized by sol-gel polymerization of titanium tetraisopropoxide using dialysis [3]. Titania-silica $\left(\mathrm{TiO}_{2}-\mathrm{SiO}_{2}\right)$ cryogel microhoneycombs are successfully prepared through unidirectional freezing and subsequent freeze drying 
of $\mathrm{TiO}_{2}-\mathrm{SiO}_{2}$ hydrogels, which are synthesized by the two-step hydrolysis of titanium tetraisopropoxide and tetramethylorthosilicate and the sol-gel polymerization [15]. The microfibers and microhoneycombs show higher photocatalytic activities for decomposition of large-organic molecules than a commercial photocatalyst, Degussa P-25. Alumina-silica $\left(\mathrm{Al}_{2} \mathrm{O}_{3}-\mathrm{SiO}_{2}\right)$ microfibers and microheoneycoms are obtained by applying ice-templating to the mixed metal oxide hydrogels of $\mathrm{Al}_{2} \mathrm{O}_{3}-\mathrm{SiO}_{2}$, which are prepared through sol-gel synthesis technique [16]. The materials have Brønsted acid sites, which allow them to act as acid catalysts. Carbon cryogel microfibers and microhoneycombs are prepared by applying ice-templating to resorcinol and formaldehyde $[4,17]$. Although the freezing operation greatly decreases their porosities, $\mathrm{CO}_{2}$ activation is extremely useful for improving the porosity because the honeycomb structure can be maintained during activation [18].

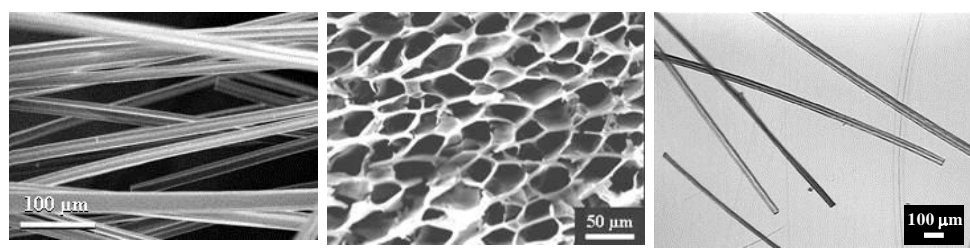

(a) Titania fiber

(b) Titania-silica microhoneycomb (c) Alumina-silica fiber

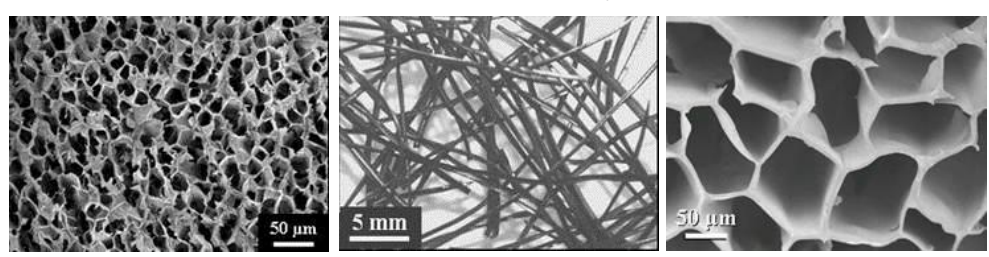

$\begin{array}{lll}\text { (d) Alumina-silica microhoneycomb } & \text { (e) Carbon fiber } & \text { (f) Carbon microhoneycomb }\end{array}$

Fig. 5. SEM images of porous materials prepared by ice-templating.

\section{Foam Material Preparation from a Suspension of Carbon Nanotubes}

Freezing induces a pattern formation in a colloidal suspension when the propagation velocity of the freezing front is kept at a certain value [19]. Microstructure of frozen colloidal suspension cannot be obtained by simply replacing water in unfrozen solution by ice. The freezing front rejects suspended particles when the front velocity is sufficiently low. However, it engulfs the particles when the velocity is sufficiently high. Physics of this particle rejection process theoretically reveals that the interfacial premelting phenomenon controls the behavior of a foreign particle at the solidifying interface [20-22]. When the solidifying interface encounters a stationary particle, repulsive intermolecular interaction between the solid and the particle creates a thin liquid layer to reduce the total free energy at the interface. This premelting layer formation allows a particle to move away from the freezing front by repulsive interaction with the ice (Fig. 6). The repulsive force is a dominating factor that controls the particle movement kinetics. Simply stated, a particle is rejected by the ice front when the particle mobility velocity is higher than the ice front velocity; otherwise the particle is engulfed. As a consequence, suspended particles are sometimes rejected and sometimes engulfed in a frozen bulk to yield complex microstructure. 


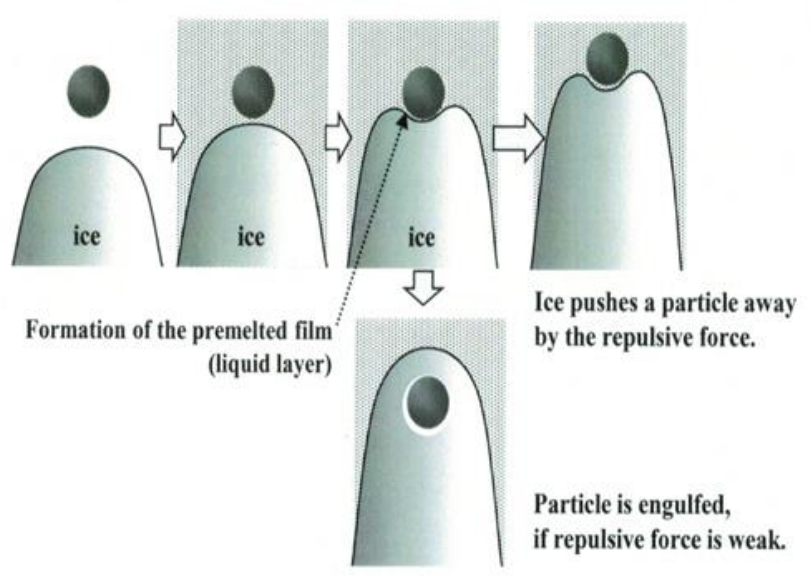

Fig. 6. Schematic illustration of the pre-melt layer formation at the ice interface.

CNT foams were prepared from an aqueous suspension of CNTs dispersed by chitosan. In the experiment, the sample solution was set on a cooling plate and frozen unidirectionally according to a selected cooling protocol. The frozen sample thus obtained was consecutively freeze-dried on the same cooling shelf. The microstructures of the resulting CNT foams were depicted in Fig. 7. These images confirm that slender macropores were formed with a particular regularity. A typical microstructure obtained via contact freezing was a bundle of cylindrical pores. A layered monolith was also prepared via a suitable freezing protocol. By enlarging the matrix of the pore wall, it was found to be composed of CNTs. However, it should be noted that the architectures are maintained by chitosan, a biopolymer that helps CNTs disperse in the original solution. The sponge-like elastic feature of the typical CNT foam specimen could be clearly observed. The foam materials prepared by the present method were highly porous, with a porosity of about $80-98 \%$ and a bulk density of $0.05-0.06 \mathrm{~g} / \mathrm{cm}^{3}$ dependent on the original formulation.

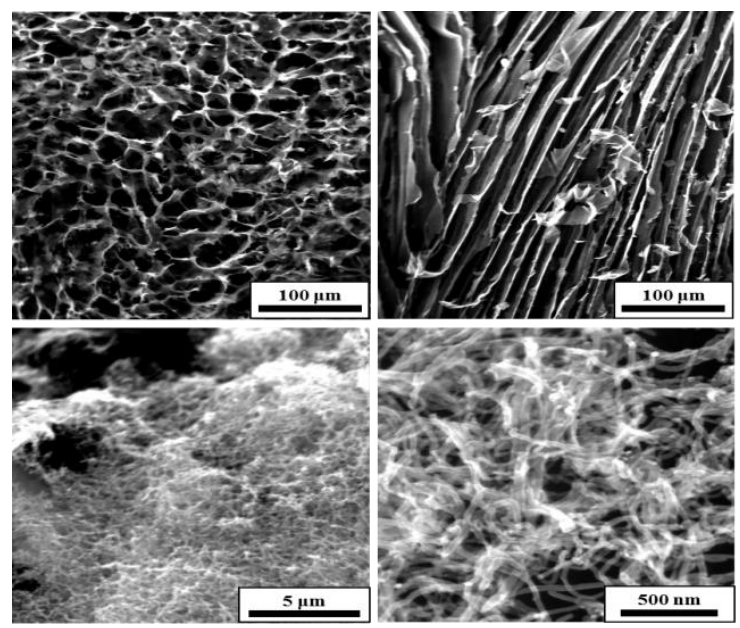

Fig. 7. SEM images of CNT foams.

\section{Application of CNT Foam as a Sensing Probe}

The foam specimens prepared in this work showed low electrical resistivity in the range of $1-10 \mathrm{k} \Omega / \mathrm{m}$. This is of course derived from interconnected CNTs in the foam. A foam specimen attached to electrical probes was placed in a vacuum chamber, and pressure inside the chamber and electrical resistivity were monitored with a pressure transmitter and digital multimeter, respectively. As shown in Fig. 8, the resistivity responded to the rise and drop in the pressure. This relationship may be attributed to the change in electrical resistivity due to the adsorption and desorption of gases on the foam walls composed of CNTs. The rapidity of the response and the absolute resistivity value of a CNT foam was found to depend on the preparation condition. Thongprachan et al. [8] reported that the absolute resistivity value of a CNT foam 
decreased with increasing CNT content, and so did the specific value of resistivity difference. The quality and quantity of contacts between the CNTs in the bulk walls were influenced by the CNT concentration, and a difference in the former appeared as a difference in the electrical resistance of the specimen.

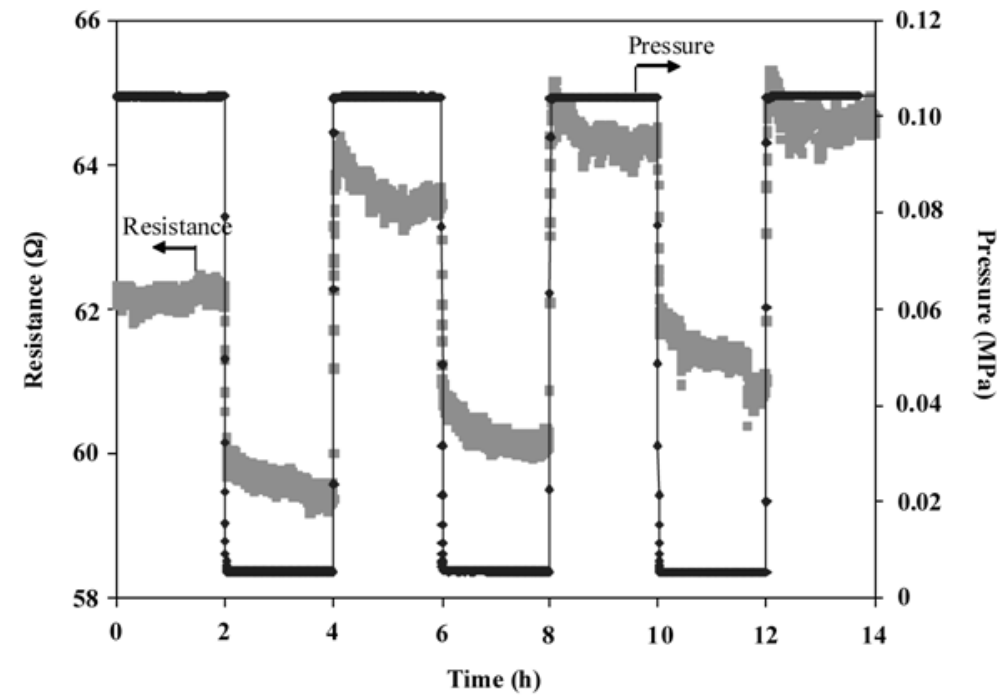

Fig. 8. Relationship between electrical resistivity and gas pressure for CNT foam.

Nakagawa et al. [23] recently applied CNT foams to gas diffusion layers (GDLs) for a proton exchange membrane fuel cell (PEMFC). They demonstrated that the fuel cell performances were comparable to one prepared with conventional carbon paper. The CNT foams were useful in reducing the film resistances of the PEMFCs, probably owing to the elasticity and specific microstructure of the prepared CNT foams. Interestingly, the microstructure of the prepared carbon foams affected the cell performance, and therefore the interconnected carbon networks formed during the freezing process were closely linked to the cell performance. The freezing process is considered to control the degree of overlap among those CNTs that influence the electrochemical properties in the fuel cell. It may be considered that ice-templating controls the nanostructures created by CNT arrays.

\section{Conclusions}

Two similar but slightly different applications of the ice-templating yielded a variety of organic and inorganic porous materials whose morphology could range from lamella, flat fiber, microhoneycomb and polygonal fiber, and whose microstructure consisted of adjustable micro-/meso-porosity as well as macroporous foam. The constructed correlations of the average macropore size $\left(d_{\mathrm{mac}}\right)$ of silica gel microhoneycombs (SMHs) to the freezing condition as well as the average wall thickness of SMHs to $C_{\mathrm{s}} d_{\mathrm{mac}}$, a product of the silica solution initial concentration and $d_{\mathrm{mac}}$, are handy for microstructure design of SMHs. Macroporous CNT foams were also prepared from aqueous suspensions of CNTs and applied as a sensing probe for gas pressure or as the gas diffusion layers of PEMFC with lower film resistance.

\section{References}

[1] S. R. Mukai, H. Nishihara, and H. Tamon, "Porous properties of silica gels with controlled morphology synthesized by unidirectional freeze-gelation," Micropor. Mesopor. Mater., vol. 63, pp. 43-51, 2003.

[2] S. R. Mukai, H. Nishihara, and H. Tamon, "Formation of monolithic silica gel microhoneycombs (SMHs) using pseudosteady state growth of microstructural ice crystals," Chem. Commun., pp. 874-875, 2004.

[3] S. R. Mukai, H. Nishihara, S. Shichi, and H. Tamon, "Preparation of porous $\mathrm{TiO}_{2}$ cryogel fibers through unidirectional freezing of hydrogel followed by freeze-drying," Chem. Mater., vol. 16, pp. 4987-4991, 2004. 
[4] H. Nishihara, S. R. Mukai, and H. Tamon, "Preparation of resorcinol-formaldehyde carbon cryogel microhoneycombs," Carbon, vol. 42, pp. 899-901, 2004.

[5] S. R. Mukai, H. Nishihara, and H. Tamon, "Morphology maps of ice-templated silica gels derived from silica hydrogels and hydrosols," Micropor. Mesopor. Mater., vol. 116, pp. 166-170, 2008.

[6] K. Nakagawa, "Foam materials made from carbon nanotubes," Carbon Nanotubes, InTech, pp. 313-334, 2011.

[7] C. Lau, M. J. Cooney, and P. Atanassov, "Conductive macroporous composite chitosan-carbon nanotube scaffolds," Langmuir, vol. 24, pp. 7004-7010, 2008.

[8] N. Thongprachan, K. Nakagawa, N. Sano, T. Charinpanitkul, and W. Tanthapanichakoon, "Preparation of macroporous solid foam from muti-walled carbon nanotubes by freeze-drying technique," Mater. Chem. Phys., vol. 112, pp. 262-269, 2008.

[9] S. M. Kwon, H. S. Kim, and H. J. Jin, "Multiwalled carbon nanotube cryogels with aligned and nonaligned porous structures," Polymer, vol. 50, pp. 2786-2792, 2009.

[10] I. Olivas-Armendariz, P. Garcia-Casillasa, R. Martiinez-Sanchez, A. Martiinez-Villafane, and C. A. Martinez-Perez, "Chitosan/MWCNT composites prepared by thermal induced phase separation," J. Alloys Compd., vol. 495, pp. 592-595, 2010.

[11] K. Nakagawa, N. Thongprachan, T. Charinpanitkul, and W. Tanthapanichakoon, "Ice crystal formation in the carbon nanotube suspension: A modelling approach," Chem. Eng. Sci., vol. 65, pp. 1438-1451, 2010.

[12] C. M. Leroy, F. Carn, R. Backov, M. Trinquecoste, and P. Delhaes, "Multiwalled carbon nanotubebased carbon foams," Carbon, vol. 45, pp. 2307-2320, 2007.

[13] M. A. Worsley, S. O. Kucheyev, J. H. Satcher, A. V. Hamza, and T. F. Baumann, "Mechanically robust and electrically conductive carbon nanotube foams," Applied Physics Letters, vol. 94, p. 073115, 2009.

[14] H. Nishihara, S. R. Mukai, D. Yamashita, and H. Tamon, "Ordered macroporous silica by icetemplating," Chem. Mater., vol. 17, pp. 683-689, 2005.

[15] H. Nishihara, S. R. Mukai, and H. Tamon, "Preparation of titania-silica cryogels with controlled shapes and photocatalysis through unidirectional freezing," Mater. Lett., vol. 64, pp. 959-961, 2010.

[16] H. Nishihara, S. R. Mukai, Y. Fujii, T. Tago, T. Masuda, and H. Tamon, "Preparation of monolithic $\mathrm{SiO}_{2}-\mathrm{Al}_{2} \mathrm{O}_{3}$ cryogels with inter-connected macropores through ice templating," J. Mater. Chem., vol. 16, pp. 3231-3236, 2006.

[17] S. R. Mukai, H. Nishihara, T. Yoshida, K. Taniguchi, and H. Tamon, "Morphology of resorcinolformaldehyde gels obtained through ice-templating," Carbon, vol. 43, pp. 1563-1565, 2005.

[18] H. Tamon, M. Sakamoto, T. Suzuki, and N. Sano, "Preparation of carbon cryogel microhoneycomb by ice-templating and activation," Proc. 17th International Drying Symposium (IDS 2010), Magdeburg, Germany, 2010.

[19] S. Deville, E. Saiz, and A. Tomsia, "Ice-templated porous alumina structures," Acta Materialia, vol. 55, pp. 1965-1974, 2007.

[20] J. G. Dash, H. Fu, and J. S. Wettkaufer, "The premelting of ice and its environmental consequences," Rep. Prog. Phys., vol. 58, pp. 115-167, 1995.

[21] J. G. Dash, A. W. Rempel, and J. S. Wettlaufer, "The physics of premelted ice and its geophysical consequences," Rev. Mod. Phys., vol. 78, pp. 695-741, 2006.

[22] J. S. Wettlaufer, and M. G. Worster, "Premelting dynamics," Annu. Rev. Fluid Mech., vol. 38, pp. 427452, 2006.

[23] K. Nakagawa, Y. Yasumura, N. Thongprachan, and N. Sano, "Freeze-dried solid foams prepared from carbon nanotube aqueous suspension: Application to gas diffusion layers of a proton exchange membrane fuel cell," Chem. Eng. Process., vol. 50, pp. 22-30, 2011. 